(с) О.А. Кошельская' , А.С. Сушкова'1, О.А. Журавлева', И.В. Винницкая' ', Н.Г. Бразовская², Е.С. Кравченко', Т.Е. Суслова', Р.С. Карпов

'ФГБНУ Томский национальный исследовательский медицинский центр Российской академии наук, Томск

ФГБОУ ВО Сибирский государственный медицинский университет Минздрава России, Томск

оБосновАНИЕ. Во многих рандомизированных и наблюдательных исследованиях доказана способность статинов оказывать диабетогенное и гипергликемическое воздействие, но до настоящего времени не разработаны рекомендации в отношении прогнозирования динамики гликемии при проведении липидснижающей терапии.

ЦЕЛь. Определить комбинацию факторов, обладающих прогностической значимостью в отношении характера изменений базальной гликемии в ходе полугодовой липидснижающей терапии у пациентов высокого сердечно-сосудистого риска.

МЕТОДЫ. В исследование включены 68 пациентов, в том числе 50 пациентов с сахарным диабетом (СД) или нарушенной толерантностью к глюкозе (НТГ), из которых у 29 документирована ишемическая болезнь сердца (ИБС) со стабильной стенокардией, и 18 больных ИБС без нарушений углеводного обмена. Рандомизированы 3 группы пациентов: 1-я (n=33) - на приеме аторвастатина, 2-я ( $n=17)$ - на комбинации аторвастатина с эзетимибом, 3-я (n=18) - на розувастатине. В ходе 24-недельной терапии оценивали динамику показателей липидтранспортной функции крови и метаболизма глюкозы/инсулина. Для установления возможных предикторов роста базальной гликемии использовали пошаговый дискриминантный анализ.

РЕзультАТы. Переносимость терапии была хорошей, все включенные пациенты закончили 24-недельный период лечения. Во всех группах установлен достоверный гиполипидемический эффект, половина пациентов общей группы (n=34) достигали целевого уровня холестерина липопротеинов низкой плотности (ХС ЛПНП). Если у пациентов 1-й и 3-й групп существенной динамики медианных значений базальной гликемии не происходило, то во 2-й - имел место рост базальной гликемии от 5,5 $(5,3-6,6)$ до 6,3 $(5,6-7,8)$ ммоль/л ( $p=0,0014)$, что сопровождалось увеличением значений индекса HOMA-IR ( $p=0,024)$. Возрастание базальной гликемии разной степени имело место у 48,5\%, 70,6\% и 44,4\% пациентов в группах 1-3 соответственно. В общей группе пациентов (n=68) выполнен многофакторный пошаговый дискриминантный анализ. В каноническую линейную дискриминантную функцию были включены: исходные уровни базальной глюкозы, общего холестерина (ОхС), триглицеридов (ТГ) и соотношение ХС ЛПНП/ХС ЛПВП. Чувствительность и специфичность модели составили 75\%; при прогнозировании динамики гликемии в ходе липидснижающей терапии 51 случай из 68 был классифицирован правильно.

ЗАКЛЮЧЕНИЕ. Наши данные демонстрируют возможность прогнозирования разнонаправленной динамики базальной гликемии при проведении липидснижающей терапии и позволяют идентифицировать пациентов, у которых индивидуальный риск роста гликемии натощак в ходе этой терапии может быть наиболее высоким.

кЛЮчЕВЫЕ СЛОВА: липидснижающая терапия; статины; эзетимиб; базальная гликемия; ишемическая болезнь сердца; сахарный диабет

\title{
PREDICTION OF BASAL GLYCAEMIA DYNAMICS DURING TREATMENT WITH 6-MONTH LIPID- LOWERING THERAPY IN PATIENTS AT HIGH RISK OF CARDIOVASCULAR DISEASE
}

(c) Olga A. Koshelskaya', Anastasiya S. Sushkova', Olga A. Zhuravleva', Irina V. Vinnizkaya', Nataliya G. Brazovskaya², Elena S. Kravchenko', Tatyana E. Suslova', Rostislav S. Karpov'

'Tomsk National Research Medical Center of the Russian Academy of Sciences, Tomsk, Russia

2Siberian State Medical University, Tomsk, Russia

BACKGROUND. A major meta-analysis has confirmed the ability of statins to exert both diabetogenic and hyperglycaemic effects. To date, practical recommendations for predicting glucose dynamics during lipid-lowering therapy have not been developed.

AIMS. Identify the combination of factors that can predict changes in basal glycaemia during 6-month lipid-lowering therapy in patients at high risk of cardiovascular disease.

METHODS. This study reports on 50 patients with diabetes or impaired glucose tolerance, and 18 patients with coronary artery disease without disorders of carbohydrate metabolism. Of note, 29 of the 50 diabetic or glucose intolerant patients had documented ischaemic heart disease (stable angina). Patients were randomised into three groups: $\mathrm{Gr} .1$ ( $n=33$, atorvastatin therapy), Gr.2 ( $\mathrm{n}=17$, atorvastatin in combination with ezetimibe) and Gr.3 ( $n=18$, rosuvastatin therapy). After treatment for 24 weeks, we assessed lipid profile dynamics, metabolism of glucose/insulin and the HOMA-IR index. Multivariate analysis was then performed to identify factors that predicted increases in basal glycaemia. 
RESULTS. All of the included patients completed 24 weeks of treatment ( $\mathrm{N}=68$ ). Lipid-lowering effect was significant in all three groups, and overall, target LDL cholesterol level was achieved in $50 \%$ of patients $(n=34)$. In Gr.2, basal glucose level increased from 5.5(5.3-6.6) to 6.3(5.6-7.8) $\mathrm{mmol} / \mathrm{I}(\mathrm{p}=0.0014)$, which was accompanied by an increase in HOMA-IR ( $p=0.024)$. No significant change in basal glycaemia was observed in Grs.1 and 3. Moreover, an increase in the basal glycaemia was observed in $48.5 \%$ of patients in Gr.1, 70.6\% in Gr.2 and 44.4\% in Gr.3. Multivariate discriminant analysis across all patient groups revealed a canonical linear discriminant function that included the following factors: baseline basal glucose levels, total cholesterol levels, triglycerides and ratio of LDL/HDL cholesterol. Sensitivity and specificity of the model accounted for 75\%; 51 out of the 68 cases were correctly classified when predicting the dynamics of basal glucose during lipid-lowering therapy.

CONCLUSIONS. Our data demonstrate the ability to predict the dynamics of the basal glycaemia during lipid-lowering therapy. This may allow for a new way to identify patients at high risk of statin-related increases in glycaemia.

KEYWORDS: lipid-lowering therapy; statins; ezetimibe; basal glycemia; coronary artery disease; diabetes mellitus

Назначение статинов в настоящее время является обязательным условием первичной и вторичной профилактики атеросклеротических осложнений у лиц с высоким и очень высоким кардиоваскулярным риском вне зависимости от исходного уровня холестерина липопротеинов низкой плотности (ХС ЛПНП), а главным критерием успешности терапии считается его снижение не менее, чем на 50\% [1, 2]. Вместе с тем доказано, что терапия статинами может оказывать нежелательные диабетогенный и гипергликемический эффекты, которые имеют дозозависимый характер [3], демонстрируют прямую связь с возрастом пациентов [3], полом, расой [4, 5], видом сопутствующей терапии [6] и наиболее часто реализуются у пациентов с наличием исходных метаболических факторов риска [3]. Одним из рекомендованных подходов к снижению ХС ЛПНП до целевого уровня является назначение комбинации статинов с ингибитором кишечной абсорбции эзетимибом, что позволяет достигать интенсивного снижения уровня ХС ЛПНП при использовании средних доз статинов $[1,2,7,8]$ и теоретически может снизить риск их потенциальных нежелательных эффектов. Однако до настоящего времени влияние комбинированной липидснижающей терапии на обмен глюкозы и инсулина изучено недостаточно [8-11]. Не разработаны практические рекомендации по оценке индивидуального риска ассоциированных с проведением липидснижающей терапии нежелательных изменений обмена глюкозы/ инсулина, что потенциально способно снизить эффективность положительного воздействия статинов на сердечно-сосудистый прогноз [11].

\section{ЦЕЛЬ}

Изучить динамику показателей метаболизма глюкозы/ инсулина в ходе 24-недельной липидснижающей терапии при использовании разных схем снижения ХС ЛПНП у пациентов высокого сердечно-сосудистого риска и определить комбинацию факторов, способных прогнозировать характер изменений базальной гликемии в процессе этой терапии.

\section{МЕТОДЫ}

\section{ДИЗАЙН ИССЛЕДОВАНИЯ}

В исследование были включены 68 пациентов высокого и очень высокого сердечно-сосудистого риска. На первом этапе в ходе открытого проспективного не- рандомизированного исследования были изучены липидные и метаболические эффекты аторвастатина $(n=33)$, на втором этапе в ходе открытого сравнительного проспективного рандомизированного исследования вновь включенных 35 пациентов оценивались липидные и метаболические эффекты комбинации аторвастатина с эзетимибом ( $n=17)$ и розувастатина $(n=18)$. Распределение пациентов на разные схемы терапии в ходе второго этапа проводилось методом конвертов. На третьем этапе исследования для многомерной оценки прогностической значимости признаков, предсказывающих изменения базальной гликемии в ходе липидснижающей терапии, в общей группе пациентов $(\mathrm{n}=68)$ был выполнен пошаговый дискриминантный анализ.

\section{Критерии соответствия}

Критерии включения в исследование: пациенты высокого и очень высокого сердечно-сосудистого риска, нуждающиеся в проведении длительной терапии статинами: больные ишемической болезнью сердца (ИБС) со стабильной стенокардией I-II ФК без нарушений углеводного обмена; пациенты с сахарным диабетом типа 2 (СД2), нарушением толерантности к глюкозе (НТГ) или повышением гликемии натощак (ПГТ) и документированной ИБС со стабильной стенокардией I-ІІ ФК либо с высоким риском ее развития и наличием поражений органов-мишеней в возрасте 40-75 лет.

В исследование не включались пациенты с гипертриглицеридемией более 4,5 ммоль/л, СД 1 типа, заболеваниями печени в активной фазе, вторичной дислипидемией (ДЛП) на фоне заболеваний почек, при наличии беременности или в периоде лактации, имеющие противопоказания к назначению статинов. Критериями исключения являлись: индивидуальная непереносимость, возрастание трансаминаз или креатинфосфокиназы на фоне терапии (выше трех верхних границ нормы), развитие миастении или миалгии, выраженное прогрессирование сопутствующей патологии, отказ больного от участия в исследовании.

\section{Условия проведения}

Исследование было проведено на базе отделения атеросклероза и хронической ИБС (Научно-исследовательский институт кардиологии, ФГБНУ «Томский национальный исследовательский медицинский центр Российской академии наук») - пациенты наблюдались амбулаторно, посещали центр для проведения запланированных визитов, диагноз основного и фонового заболеваний у них был верифицирован ранее. 
Продолжительность исследования

Продолжительность наблюдения составила 24 нед. Визиты пациентов проводились до начала лечения, через 4, 12, 24 нед терапии. В ходе исследования все запланированные временные интервалы по визитам больных были соблюдены.

\section{Описание медицинского вмешательства}

Забор крови производился больным до начала терапии и через 4, 12 и 24 нед лечения. Целевым уровнем ХС ЛПНП на момент начала проведения исследования считали его снижение менее 2,0 ммоль/л, у больных СД без установленной ИБС - менее 2,5 ммоль/л [12].

У пациентов на монотерапии аторвастатином (Аторис, KRKA, Словения) лечение начинали с дозы 10 мг/сут, затем, при отсутствии достижения целевого уровня ХС ЛПНП, доза увеличивалась до 20 мг/сут, через 3 мес терапии при необходимости - до 40 мг/сут. В группе комбинированной терапии использовали аторвастатин в дозе 10-20 мг/сут и эзетимиб 10 мг/сут (Эзетрол, Schering-Plough Products, Бельгия). У пациентов на монотерапии розувастатином (Мертенил, Гедеон Рихтер, Венгрия) его начальная доза в первый месяц лечения составила 10 мг/сут, затем, при отсутствии достижения целевого уровня ХС ЛПНП, доза увеличивалась до 20 мг/сут.

\section{Основной исход исследования}

Определение совокупности факторов, обладающих прогностической значимостью в отношении различной динамики базальной гликемии при проведении 24-не- дельной липидснижающей терапии у пациентов высокого и очень высокого сердечно-сосудистого риска.

\section{Дополнительные исходы исследования}

Оценка гиполипидемической эффективности и метаболических эффектов трех схем терапии, основанных на титрации дозы розувастатина, аторвастатина и комбинации аторвастатина с эзетимибом у пациентов высокого и очень высокого риска сердечно-сосудистых осложнений.

\section{Анализ в подгруппах}

Анализ липидных и метаболических эффектов проводился в трех группах пациентов, получавших терапию аторвастатином (группа 1, n=33), комбинацией аторвастатина с эзетимибом (группа 2, n=17) и розувастатином (группа 3, n=18). Для проведения следующего этапа исследования пациенты трех групп были объединены в общую группу (n=68). Сравнительная клиническая характеристика больных, включенных в исследование, представлена в табл. 1. Существенных межгрупповых различий характера текущей базовой и сахароснижающей терапии не было, в течение всего периода исследования их схемы не менялись.

Методы регистрации исходов

До начала лечения, через 4, 12 и 24 нед терапии определяли содержание общего холестерина (ОХC), холестерина липопротеинов высокой плотности (ХС ЛПВП), ХС ЛПНП, триглицеридов крови (ТГ). В исходе и по окончанию исследования анализировали концентрации аполипопротеина A1 (апоА1) и аполипопротеина В (апоВ) в сыворотке

Таблица 1. Клиническая характеристика включенных в исследование больных

\begin{tabular}{|c|c|c|c|}
\hline Показатель & $\begin{array}{c}\text { Группа } 1 \\
(\mathrm{n}=33)\end{array}$ & $\begin{array}{c}\text { Группа } 2 \\
(\mathrm{n}=17)\end{array}$ & $\begin{array}{c}\text { Группа } 3 \\
(\mathrm{n}=18)\end{array}$ \\
\hline Пол (мужчины/женщины), \% & $12 / 21(36,4 / 63,6)$ & $9 / 8(52,9 / 47,1)$ & $8 / 10(44,4 / 55,6)$ \\
\hline Возраст, лет* & $59,6 \pm 6,1$ & $62,3 \pm 6,0$ & $62,2 \pm 6,3$ \\
\hline $\begin{array}{l}\text { Доля пациентов с СД2, НТГ и повышением гликемии } \\
\text { натощак, \% }\end{array}$ & 75,8 & 64,7 & 77,8 \\
\hline Доля больных с ИБС, \% & 54,5 & 88,2 & 77,8 \\
\hline Доля больных с ГБ, \% & 93,9 & 76,5 & 100 \\
\hline Офисное систолическое АД, мм рт.ст.* & $131,8 \pm 14,8$ & $120,7 \pm 13,5$ & $126,0 \pm 17,1$ \\
\hline Офисное диастолическое АД, мм рт.ст.* & $82,1 \pm 9,4$ & $72,2 \pm 7,2$ & $73,2 \pm 7,4$ \\
\hline $\mathrm{HbA}_{1 c^{\prime}} \%^{\#}$ & $6,60(6,1-7,3)$ & $6,47(6,1-7,0)$ & $6,65(5,8-8,4)$ \\
\hline Индекс массы тела, кг/м²* & $31,1 \pm 4,4$ & $28,6 \pm 3,5$ & $30,0 \pm 4,9$ \\
\hline Доля пациентов с ожирением (ИМТ > 30, кг/м²), \% & 57,6 & 41 & 50 \\
\hline Окружность талии/окружность бедер* & $0,94 \pm 0,07$ & $0,95 \pm 0,07$ & $0,96 \pm 0,08$ \\
\hline Доля пациентов с каротидным атеросклерозом, \% & 78,8 & 76,5 & 83,3 \\
\hline \multicolumn{4}{|l|}{ Доля пациентов, принимающих: } \\
\hline блокаторы PAAC, \% & 87,9 & 70,6 & 77,8 \\
\hline ß-адреноблокаторы, \% & 60,6 & 64,7 & 88,9 \\
\hline антагонисты кальция, \% & 51,5 & 29,4 & 44,4 \\
\hline тиазидные диуретики, \% & 32,6 & 23,5 & 27,8 \\
\hline \multicolumn{4}{|l|}{ Доля пациентов, совместно принимающих: } \\
\hline $\beta$-адреноблокаторы土диуретики, \% & 23,6 & 17,6 & 22,2 \\
\hline
\end{tabular}

Примечания: ИБС - ишемическая болезнь сердца; ГБ - гипертоническая болезнь; АД - артериальное давление; НbА

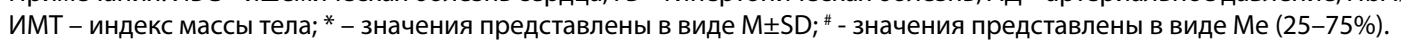


крови и вычисляли соотношение апоВ/апоА1, а также рассчитывали содержание ХС не-ЛПВП (ОХС-ХС ЛПВП), отношение ХС ЛПНП/ХС ЛПВП и индекс атерогенности: (ОХС-ХС ЛПВП)/ХС ЛПВП.

У всех пациентов в процессе терапии оценивали уровень гликированного гемоглобина $\left(\mathrm{HbA}_{1 c}\right)$, базальной и постпрандиальной гликемии и концентрацию С-пептида в крови; у пациентов, не получавших терапию инсулином, определяли также уровень в крови базального и постпрандиального инсулина и рассчитывали индекс инсулинорезистентности HOMA-IR: $\left[\left(I_{0}\right)^{*}\left(G_{0}\right)\right] / 22,5$, где I - базальная гликемия (ммоль/л), G - базальная инсулинемия (мкME/ мл).

\section{Этическая экспертиза}

Исследование было выполнено в соответствии со стандартами надлежащей клинической практики (Good Clinical Practice) и принципами Хельсинкской Декларации. Протокол исследования был одобрен Локальным этическим комитетом НИИ кардиологии Томского НИМЦ (протокол №139 от 18.11.2015). До включения в исследование у всех участников было получено письменное информированное согласие.

\section{Статистический анализ}

Принципы расчета размера выборки: при построении многомерных классификационных моделей руководствовались известным правилом: на одну переменную в модели должно приходиться не менее десяти наблюдений.

Memodы статистического анализа данных: статистический анализ материала проводился с использованием пакета прикладных программ Statistica 10.0. Проводилась проверка нормальности распределения количественных признаков, для описания признаков с нормальным распределением использовали среднее с указанием стандартного отклонения, для признаков с отличным от нормального распределения указывали медиану и межквартильный размах - 25-й и 75-й процентили. Сравнение количественных признаков проводили по критерию Манна-Уитни, сравнение качественных - с использованием таблиц сопряженности 2х2 по критерию хи-квадрат Пирсона с поправкой Йетса и точному критерию Фишера. Для оценки взаимосвязи признаков рассчитывали коэффициент ранговой корреляции Спирмена (Rs). Для многомерной оценки прогностической значимости признаков, предсказывающих рост гликемии, использовался дискриминантный пошаговый анализ. Результаты статистического анализа принимались как статистически значимые при р<0,05.

\section{РЕЗУЛЬТАТЫ}

\section{Объекты (участники) исследования}

В исследование были включены 68 пациентов высокого и очень высокого сердечно-сосудистого риска, в том числе 18 больных ИБС со стабильной стенокардией не выше II ФК без нарушений углеводного обмена и 50 пациентов с СД2, НТГ или ПГТ, из которых у 29 пациентов была документирована ИБС со стабильной стенокардией I-ІІ ФК

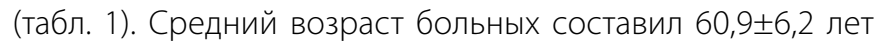
(м/ж 29/39), уровень артериального давления (АД) на фоне текущей базовой терапии не превышал 140/90 мм рт.ст.
Основные и дополнительные результаты

\section{исследования}

Через 24 нед лечения средние оттитрованные дозы составили: для аторвастатина в группах 1 и 2 - 13,6 мг/сут и 13,5 мг/сут соответственно, для розувастатина в группе 3 - 12,5 мг/сут, при этом половина пациентов высокого и очень высокого сердечно-сосудистого риска в нашем исследовании достигали целевого уровня ХС ЛПНП.

Динамика показателей липидтранспортной функции крови в ходе терапии представлена в таблицах 2 и 3. Во всех трех группах и общей группе пациентов установлен достоверный гиполипидемический эффект в виде снижения уровней ОХС, ХС ЛПНП, ХС не-ЛПВП, ТГ, отношения ХС ЛПНП/ХС ЛПВП и индекса атерогенности через 24 нед лечения. В группе комбинированной липидснижающей терапии имело место повышение уровня ХС ЛПВП на 18\% ( $p=0,015)$, что, однако, не сопровождалось приростом содержания апоА1 в сыворотке крови. В общей группе пациентов, а также в группах 2 и 3 имело место достоверное снижение соотношения апоВ/апоА1, что происходило за счет уменьшения содержания апоВ. На фоне монотерапии аторвастатином снижение содержания апоВ не сопровождалось существенными изменениями отношения апоВ/апоА1.

Динамика показателей углеводного обмена на фоне использованных режимов липидснижающей терапии представлена в табл. 4. Статистически значимой динамики содержания $\mathrm{HbA}_{1 с}$ в течение полугодового периода лечения не происходило ни в одной из групп пациентов. Тем не менее, на фоне комбинированной терапии аторвастатином и эзетимибом наблюдался достоверный рост уровня базальной гликемии от 5,5 $(5,3-6,6)$ ммоль/л до $6,3(5,6-7,8)$ ммоль/л $(p=0,0014)$, что сопровождалось возрастанием индекса HOMA-IR $(p=0,024)$. Хотя при титрации доз аторвастатина и розувастатина существенных изменений средних значений базальной гликемии не происходило, в ходе индивидуального анализа установлено, что возрастание концентрации глюкозы натощак разной степени все же имело место во всех лечебных группах: у 8 (44,4\%) больных в группе 3, у 16 (48,5\%) - в группе 1 и у $12(70,6 \%)$ пациентов в группе 2.

Поскольку при сравнении основных клинических данных пациентов всех трех групп до начала терапии каких-либо существенных межгрупповых различий обнаружено не было, следующим этапом нашего исследования стал поиск комбинации факторов, способных предопределить реализацию нежелательных эффектов липидснижающей терапии в отношении роста гликемии.

С этой целью в общей группе пациентов $(\mathrm{n}=68)$, среди которых у 36 наблюдался рост базальной гликемии в ходе терапии, мы отобрали 24 клинико-лабораторных показателя, потенциально ассоциированных с разнонаправленной динамикой базальной гликемии. Статистически значимые межгрупповые различия продемонстрировали следующие показатели: ОХС, ТГ, ХС ЛПНП, отношение ХС ЛПНП/ХС ЛПВП, уровень базальной глюкозы. В силу того, что ОХС и ХС ЛПНП имели сильную, близкую к линейной, корреляционную взаимосвязь с уровнем базальной гликемии после терапии (Rs=0,941, p<0,001), включение обоих признаков являлось нецелесообразным. Мы использовали пошаговый многофакторный дискриминантный анализ, в котором в качестве зависимой переменной рас- 
Таблица 2. Динамика показателей липидтранспортной функции крови в общей группе и в ходе терапии аторвастатином (группа 1), комбинированной терапии аторвастатином и эзетимибом (группа 2) и розувастатином (группа 3) (приведена концентрация в ммоль/л, указаны Mediana (Q25; Q75))

\begin{tabular}{|c|c|c|c|c|c|}
\hline Липиды & & $\begin{array}{c}\text { Общая группа } \\
(n=68)\end{array}$ & $\begin{array}{c}\text { Группа } 1 \\
(\mathrm{n}=33)\end{array}$ & $\begin{array}{c}\text { Группа } 2 \\
(n=17)\end{array}$ & $\begin{array}{c}\text { Группа } 3 \\
(\mathrm{n}=18)\end{array}$ \\
\hline \multirow{3}{*}{ OXC } & исходно & $6,5(5,81 ; 7,18)$ & $6,55(5,87 ; 7,26)$ & $6,65(5,67 ; 7,13)$ & $6,31(5,53 ; 7,05)$ \\
\hline & 24 нед & $4,12(3,64 ; 4,58)^{* *}$ & $4,33(4,09 ; 4,72)^{* *}$ & $3,93(3,57 ; 4,25)^{* *}$ & $3,79(3,39 ; 4,21)^{* *}$ \\
\hline & $\triangle \%$ & $-37,3(-43,8 ;-28,1)$ & $-34,2(-40,6 ;-27,9)$ & $-40,1(-48,2 ;-28,9)$ & $-37,5(-45,4 ;-34,1)$ \\
\hline \multirow{3}{*}{ ТГ } & исходно & $1,95(1,49 ; 2,56)$ & $1,94(1,53 ; 2,56)$ & $2,06(1,56 ; 2,56)$ & $1,55(1,17 ; 2,08)$ \\
\hline & 24 нед & $1,31(1,0 ; 1,71)^{* *}$ & $1,52(1,05 ; 2,03) * *$ & $1,1(0,88 ; 1,32) * *$ & $1,3(0,97 ; 1,56)^{*}$ \\
\hline & $\triangle \%$ & $-31,2(-43,5 ;-8,0)$ & $-20,4(-43,0 ;-7,9)$ & $-43,0(-54,9 ;-35,6)$ & $-26,0(-36,2 ; 0)$ \\
\hline \multirow{3}{*}{ ХС ЛПВП } & исходно & $1,18(1,01 ; 1,29)$ & $1,22(1,1 ; 1,3)$ & $1,1(0,89 ; 1,27)$ & $1,14(1,0 ; 1,29)$ \\
\hline & 24 нед & $1,19(1,03 ; 1,44)$ & $1,19(1,05 ; 1,42)$ & $1,31(1,05 ; 1,47)^{*}$ & $1,09(1,02 ; 1,37)$ \\
\hline & $\triangle \%$ & $4,6(-8,7 ; 15,8)$ & $0(-9,0 ; 10,8)$ & $18,0(5,9 ; 37,8)$ & $-0,9(-9,7 ; 11,6)$ \\
\hline \multirow{3}{*}{ ХС ЛПНП } & ИСХОДНО & $4,4(3,79 ; 5,03)$ & $4,38(3,7 ; 5,12)$ & $4,68(3,97 ; 4,86)$ & $4,2(3,78 ; 4,73)$ \\
\hline & 24 нед & $2,17(1,86 ; 2,65)^{* *}$ & $2,43(2,06 ; 2,79)^{* *}$ & $2,02(1,86 ; 2,58)^{* *}$ & $1,96(1,7 ; 2,16)^{* *}$ \\
\hline & $\triangle \%$ & $-50,3(-57,9 ;-42,1)$ & $-46,6(-51,6 ;-38,7)$ & $-58,4(-60,5 ;-46,8)$ & $-53,4(-56,9 ;-48,8)$ \\
\hline \multirow{3}{*}{ ХС ЛПНП/ХС ЛПВП } & ИСХОДНО & $3,8(3,03 ; 4,31)$ & $3,58(3,0 ; 4,2)$ & $4,09(3,26 ; 5,16)$ & $3,61(2,97 ; 4,26)$ \\
\hline & 24 нед & $1,79(1,4 ; 2,12)^{* *}$ & $1,94(1,49 ; 2,45)^{* *}$ & $1,76(1,31 ; 2,02)^{* *}$ & $1,59(1,39 ; 2,02)^{* *}$ \\
\hline & $\triangle \%$ & $-49,9(-60,8 ;-40,6)$ & $-44,8(-53,5 ;-38,6)$ & $-62,8(-68,8 ;-50,0)$ & $-50,1(-58,7 ;-45,8)$ \\
\hline
\end{tabular}

Примечание: *; р<0,05; **; p<0,001 достоверность различий на фоне терапии в сравнении с исходными показателями. Охс; общий холестерин; ТГ; триглицериды крови; ХС ЛПВП; холестерин липопротеинов высокой плотности; ХС лПНП; холестерин липопротеинов низкой плотности.

Таблица 3. Динамика индекса атерогенности, уровня ХС не-ЛПВП и апобелков в общей группе и в ходе терапии аторвастатином (группа 1), комбинированной терапии аторвастатином и эзетимибом (группа 2) и розувастатином (группа 3) (Me (Q25; Q75))

\begin{tabular}{|c|c|c|c|c|c|}
\hline Липиды & & $\begin{array}{c}\text { Общая группа } \\
(n=68)\end{array}$ & $\begin{array}{c}\text { Группа } 1 \\
(\mathrm{n}=33)\end{array}$ & $\begin{array}{c}\text { Группа } 2 \\
(n=17)\end{array}$ & $\begin{array}{c}\text { Группа } 3 \\
(\mathrm{n}=18)\end{array}$ \\
\hline $\begin{array}{l}\text { Индекс } \\
\text { атерогенности }\end{array}$ & $\begin{array}{l}\text { исходно } \\
24 \text { нед } \\
\triangle \%\end{array}$ & $\begin{array}{c}4,52(3,78 ; 5,48) \\
2,35(1,83 ; 2,77)^{* *} \\
-46,0(-58,4 ;-37,4)\end{array}$ & $\begin{array}{c}4,5(3,57 ; 4,97) \\
2,52(2,06 ; 3,08)^{* *} \\
-44,0(-51,7 ;-36,2)\end{array}$ & $\begin{array}{c}5,14(3,88 ; 6,68) \\
2,22(1,69 ; 2,52)^{* *} \\
-61,1(-67,3 ;-41,6)\end{array}$ & $\begin{array}{c}4,4(3,61 ; 5,47) \\
2,01(1,72 ; 2,73)^{* *} \\
-48,6(-57,6 ;-41,4)\end{array}$ \\
\hline $\begin{array}{l}\text { ХС не-ЛПВП, } \\
\text { ммоль/л }\end{array}$ & $\begin{array}{l}\text { исходно } \\
24 \text { нед } \\
\triangle \%\end{array}$ & $\begin{array}{c}5,15(4,61 ; 6,0) \\
2,82(2,49 ; 3,36)^{* *} \\
-46,7(-54,2 ;-37,6)\end{array}$ & $\begin{array}{c}5,08(4,67 ; 6,04) \\
3,08(2,74 ; 3,6)^{* *} \\
-42,5(-50,0 ;-37,2)\end{array}$ & $\begin{array}{c}5,78(4,56 ; 6,05) \\
2,57(2,38 ; 3,04)^{* *} \\
-57,2(-60,2 ;-38,1)\end{array}$ & $\begin{array}{c}5,0(4,58 ; 5,72) \\
2,53(2,19 ; 3,01)^{* *} \\
-47,5(-55,3 ;-44,7)\end{array}$ \\
\hline ароA1, мг/дл & $\begin{array}{l}\text { исходно } \\
24 \text { нед } \\
\triangle \%\end{array}$ & $\begin{array}{c}163,2(130,4 ; 207,2) \\
153,67(129,1 ; 176,4) \\
-2,4(-23,4 ; 14,9)\end{array}$ & $\begin{array}{c}179,6(143,4 ; 234,2) \\
159,4(136,5 ; 228,3) \\
1,68(-36,9 ; 18,5)\end{array}$ & $\begin{array}{c}158,3(134,1 ; 173,7) \\
157,1(129,5 ; 170,33) \\
-5,6(-26,2 ; 16,0)\end{array}$ & $\begin{array}{c}140,0(128,3 ; 171,6) \\
133,7(125,0 ; 154,1) \\
-1,96(-9,24 ; 3,29)\end{array}$ \\
\hline apoB, мг/дл & $\begin{array}{l}\text { исходно } \\
24 \text { нед } \\
\triangle \%\end{array}$ & $\begin{array}{l}103,8(84,8 ; 129,3) \\
64,3(50,0 ; 86,4)^{* *} \\
-40,5(-51,3 ;-17,2)\end{array}$ & $\begin{array}{c}93,17(63,07 ; 120,9) \\
79,11(57,7 ; 96,2)^{*} \\
-23,9(-42,0 ; 12,7)\end{array}$ & $\begin{array}{l}118,9(103,7 ; 159,6) \\
65,6(52,6 ; 85,2)^{* *} \\
-47,7(-60,1 ;-37,1)\end{array}$ & $\begin{array}{l}104,6(95,9 ; 131,1) \\
60,7(48,5 ; 74,8)^{* *} \\
-43,9(-54,8 ;-35,9)\end{array}$ \\
\hline apoB/apoA1 & $\begin{array}{l}\text { исходно } \\
24 \text { нед } \\
\triangle \%\end{array}$ & $\begin{array}{c}0,65(0,46 ; 0,81) \\
0,42(0,35 ; 0,6)^{* *} \\
-31,5(-49,3 ;-7,3)\end{array}$ & $\begin{array}{c}0,48(0,42 ; 0,57) \\
0,46(0,32 ; 0,59) \\
-13,2(-34,2 ; 28,3)\end{array}$ & $\begin{array}{c}0,81(0,68 ; 0,94) \\
0,46(0,36 ; 0,57) * * \\
-40,8(-56,7 ;-31,4)\end{array}$ & $\begin{array}{c}0,75(0,69 ; 0,85) \\
0,38(0,35 ; 0,6)^{* *} \\
-44,0(-52,3 ;-29,2)\end{array}$ \\
\hline
\end{tabular}

Примечание: * ; p<0,05; ** ; р<0,001 достоверность различий на фоне терапии в сравнении с исходными показателями.

сматривали рост гликемии либо его отсутствие, а в качестве признаков-предикторов - ОХС ТГ, ХС ЛПНП/ХС ЛПВП и уровень базальной гликемии. Методом Enter (включение всех независимых переменных в модель) нами была построена модель, обладающая статистической значимостью (лямбда Вилкса 0,701, p<0,001) (табл. 5). На основании оценки нормированных коэффициентов канонической линейной дискриминантной функции (КЛДФ) (табл. 6), отражающих вклад каждой переменной в модель, была предложена диагностическая модель, основанная на формуле КЛДФ:

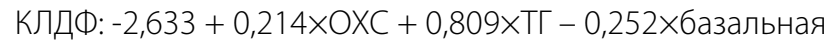
гликемия + 0,346×XС ЛПНП/ХС ЛПВП.
Диагностическая модель обладает прогностической значимостью в отношении роста базальной гликемии в ходе липидснижающей терапии у пациентов при значениях у них КЛДФ, максимально приближенных к центроиду кластера «рост гликемии есть» (его значение равно 0,607) и бо́льших точки разделения кластеров -0,038 (середина расстояния между центроидами). Сущность предложенной модели поясняется рис. 1, где изображена координатная прямая КЛДФ и центроиды КЛДФ для кластеров - «рост гликемии есть» или «роста гликемии нет». При этом центроид представляет собой значение функции, полученное при подстановке в дискриминантное уравнение средних значений предикторов в группе. Для каждого нового объ- 
Таблица 4. Динамика показателей углеводного обмена в общей группе и в ходе терапии аторвастатином (группа 1), комбинированной терапии аторвастатином и эзетимибом (группа 2) и розувастатином (группа 3) (Me (Q25; Q75))

\begin{tabular}{|c|c|c|c|c|c|}
\hline Липиды & & $\begin{array}{c}\text { Общая группа } \\
(n=68)\end{array}$ & $\begin{array}{c}\text { Группа } 1 \\
(n=33)\end{array}$ & $\begin{array}{c}\text { Группа } 2 \\
(n=17)\end{array}$ & $\begin{array}{c}\text { Группа } 3 \\
(\mathrm{n}=18)\end{array}$ \\
\hline $\begin{array}{l}\text { Базальная } \\
\text { гликемия, } \\
\text { ммоль/л }\end{array}$ & $\begin{array}{l}\text { исходно } \\
24 \text { нед } \\
\triangle \%\end{array}$ & $\begin{array}{c}6,0(5,38 ; 7,57) \\
6,35(5,46 ; 7,43) \\
1,9(-8,7 ; 16,6)\end{array}$ & $\begin{array}{l}6,17(5,61 ; 7,47) \\
5,99(5,33 ; 7,27) \\
-2,4(-11,0 ; 14,7)\end{array}$ & $\begin{array}{c}5,5(5,3 ; 6,6) \\
6,3(5,6 ; 7,8)^{* *} \\
9,02(0 ; 21,9)\end{array}$ & $\begin{array}{l}6,3(5,72 ; 8,4) \\
7,0(5,8 ; 8,5) \\
0(-11,3 ; 24,3)\end{array}$ \\
\hline $\begin{array}{l}\text { Постпрандиальная } \\
\text { гликемия, } \\
\text { ммоль/л }\end{array}$ & $\begin{array}{l}\text { исходно } \\
24 \text { нед } \\
\triangle \%\end{array}$ & $\begin{array}{c}7,17(5,8 ; 8,78) \\
7,16(5,47 ; 9,28) \\
-5,2(-19,0 ; 23,5)\end{array}$ & $\begin{array}{l}6,91(5,79 ; 8,11) \\
7,47(5,72 ; 9,26) \\
-4,6(-11,3 ; 37,6)\end{array}$ & $\begin{array}{c}7,1(5,9 ; 8,0) \\
6,4(5,2 ; 7,11) \\
-9,7(-23,4 ; 8,89)\end{array}$ & $\begin{array}{c}7,99(6,5 ; 9,75) \\
8,15(5,8 ; 10,32) \\
-8,9(-18,9 ; 16,7)\end{array}$ \\
\hline $\mathrm{HbA}_{1 c^{\prime}} \%$ & $\begin{array}{l}\text { исходно } \\
24 \text { нед } \\
\triangle \%\end{array}$ & $\begin{array}{c}6,55(6,1 ; 7,5) \\
6,40(5,9 ; 7,4) \\
0(-5,2 ; 2,7)\end{array}$ & $\begin{array}{c}6,6(6,1 ; 7,3) \\
6,53(5,95 ; 7,2) \\
0(-1,7 ; 1,9)\end{array}$ & $\begin{array}{c}6,47(6,1 ; 7,0) \\
6,40(5,8 ; 7,2) \\
2,12(-8,4 ; 17,3)\end{array}$ & $\begin{array}{c}6,65(5,8 ; 8,4) \\
6,34(5,9 ; 7,5) \\
-4,50(-19,6 ; 2,7)\end{array}$ \\
\hline $\begin{array}{l}\text { Базальный } \\
\text { С-пептид, } \\
\text { нг/мл }\end{array}$ & $\begin{array}{l}\text { исходно } \\
24 \text { нед } \\
\triangle \%\end{array}$ & $\begin{array}{c}2,26(1,88 ; 2,96) \\
2,42(1,72 ; 3,0) \\
3,00(-16,2 ; 23,4)\end{array}$ & $\begin{array}{l}2,35(2,01 ; 3,02) \\
2,42(1,72 ; 3,04) \\
4,70(-17,8 ; 17,8)\end{array}$ & $\begin{array}{c}2,09(1,92 ; 2,67) \\
2,43(1,92 ; 3,0) \\
15,20(-20,7 ; 25,1)\end{array}$ & $\begin{array}{c}2,06(1,36 ; 2,46) \\
1,94(1,56 ; 2,96) \\
-0,12(-4,78 ; 29,3)\end{array}$ \\
\hline $\begin{array}{l}\text { Постпрандиальный } \\
\text { С-пептид, } \\
\text { нг/мл }\end{array}$ & $\begin{array}{l}\text { исходно } \\
24 \text { нед } \\
\triangle \%\end{array}$ & $\begin{array}{l}4,34(3,16 ; 6,65) \\
4,96(3,35 ; 6,99) \\
7,0(-23,5 ; 73,1)\end{array}$ & $\begin{array}{l}4,78(3,33 ; 7,63) \\
5,29(4,27 ; 7,48) \\
1,30(-27,9 ; 52,7)\end{array}$ & $\begin{array}{c}4,15(2,97 ; 6,11) \\
4,92(3,75 ; 6,92) \\
16,10(-17,6 ; 91,6)\end{array}$ & $\begin{array}{c}3,91(3,14 ; 6,1) \\
3,99(2,99 ; 5,96) \\
19,10(-20,1 ; 67,8)\end{array}$ \\
\hline $\begin{array}{l}\text { Базальная } \\
\text { инсулинемия, } \\
\text { мкMЕ/мл }\end{array}$ & $\begin{array}{l}\text { исходно } \\
24 \text { нед } \\
\triangle \%\end{array}$ & $\begin{array}{c}\mathrm{n}=59 \\
9,44(6,64 ; 17,43) \\
9,24(6,74 ; 16,28) \\
10,26(-30,69 ; 37,34)\end{array}$ & $\begin{array}{c}\mathrm{n}=29 \\
12,16(7,41 ; 15,97) \\
10,93(7,59 ; 17,56) \\
8,96(-35,67 ; 24,36)\end{array}$ & $\begin{array}{c}n=16 \\
8,28(6,36 ; 9,32) \\
9,17(6,64 ; 12,03) \\
15,01(-12,65 ; 35,58)\end{array}$ & $\begin{array}{c}\mathrm{n}=14 \\
9,88(6,36 ; 18,68) \\
9,60(6,21 ; 12,42) \\
19,88(-33,51 ; 65,72)\end{array}$ \\
\hline $\begin{array}{l}\text { Постпрандиальная } \\
\text { инсулинемия, } \\
\text { мкМЕ/мл }\end{array}$ & $\begin{array}{l}\text { исходно } \\
24 \text { нед } \\
\triangle \%\end{array}$ & $\begin{array}{c}n=59 \\
23,08(13,17 ; 44,89) \\
28,65(17,11 ; 43,99) \\
42,05(-31,97 ; 145,14)\end{array}$ & $\begin{array}{c}n=29 \\
24,91(15,05 ; 62,91) \\
36,90(18,33 ; 58,92) \\
55,52(-31,97 ; 153,08)\end{array}$ & $\begin{array}{c}\mathrm{n}=16 \\
18,92(7,38 ; 32,80) \\
25,99(16,17 ; 29,91) \\
64,07(-42,22 ; 215,14)\end{array}$ & $\begin{array}{c}n=14 \\
23,46(13,17 ; 36,67) \\
32,34(12,51 ; 55,13) \\
14,62(-31,67 ; 58,99)\end{array}$ \\
\hline HOMA-IR & $\begin{array}{l}\text { исходно } \\
24 \text { нед } \\
\triangle \%\end{array}$ & $\begin{array}{c}n=59 \\
2,85(1,96 ; 4,14) \\
2,78(1,81 ; 4,60) \\
12,87(-33,64 ; 63,42)\end{array}$ & $\begin{array}{c}\mathrm{n}=29 \\
3,22(2,48 ; 4,01) \\
2,81(2,02 ; 4,67) \\
2,48(-38,34 ; 28,22)\end{array}$ & $\begin{array}{c}n=16 \\
2,08(1,67 ; 3,02) \\
2,33(2,04 ; 4,45)^{*} \\
13,2(1,72 ; 64,07)\end{array}$ & $\begin{array}{c}\mathrm{n}=14 \\
3,42(1,92 ; 6,29) \\
3,11(1,36 ; 4,33) \\
46,52(-33,64 ; 56,71)\end{array}$ \\
\hline
\end{tabular}

Примечания: * - p<0,05; ** - p<0,01 достоверность различий на фоне терапии в сравнении с исходными показателями.

екта рассчитывается значение КЛДФ и определяется соответствующая точка на координатной прямой. Объект считается принадлежащим к тому классу, чей центроид ближе к полученному значению КЛДФ.

Результаты применения классифицирующей функции к объектам из обучающей выборки продемонстрировали удовлетворительное качество классификации: коэффициент конкордации (процент правильных решений), чувствительность и специфичность прогностической модели составили 75\%, что говорит о ее достоверности и высокой эффективности в предсказании характера изменений базальной гликемии при проведении липидснижающей терапии: 51 случай из 68 был классифицирован правильно (табл. 7).

Пример 1. Пациент У., 57 лет, диагноз: ИБС: стенокардия напряжения, ФК II. Гипертоническая болезнь III стадия, риск 4. Нарушение толерантности к глюкозе. Смешанная гиперлипидемия.

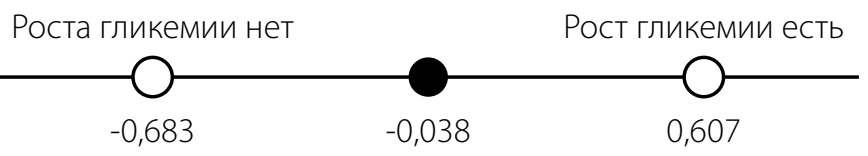

Рис. 1. Координатная прямая КЛДФ и центроиды КЛДФ для кластеров.
Липидный спектр: ОХС 6,56 ммоль/л, ТГ 2,89 ммоль/л, ХС ЛПВП 0,85 ммоль/л, ХС ЛПНП 4,38 ммоль/л, соотношение ХС ЛПНП/ХС ЛПВП 5,16. Базальная гликемия 5,3 ммоль/л, постпрандиальная гликемия 8,9 ммоль/л, С-пептид базальный 3,93 нг/мл, индекс HOMA-IR 2,1. Регулярная терапия бисопрололом, лозартаном, ацетилсалициловой кислотой. При включении в исследование рандомизирован на терапию аторвастатином 10 мг и эзетимибом 10 мг. Индивидуальное значение КЛДФ: $-2,633+0,214 \times 6,56+$ $+0,809 \times 2,89-0,252 \times 5,30+0,346 \times 5,16=1,55861$. Исходя из величины КЛДФ, прогнозировался рост базальной гликемии, что и имело место через 6 мес липидснижающей терапии, когда гликемия достигла 7,0 ммоль/л, при этом содержание постпрандиальной глюкозы, базального С-пептида и индекса HOMA-IR составили 8,9 ммоль/л, 4,5 нг/мл и 5,8 соответственно.

Пример 2. Пациентка С., 62 лет, диагноз: Гипертоническая болезнь III стадия, риск 4. ИБС: стенокардия напряжения, ФК I-ІІ. Гиперхолестеринемия. Ожирение I степени. Липидный спектр: ОХС 7,51 ммоль/л, ТГ 1,15 ммоль/л, ХС ЛПВП 1,15 ммоль/л, ХС ЛПНП 5,4 ммоль/л, соотношение ХС ЛПНП/ХС ЛПВП 3,39. Базальная гликемия 5,1 ммоль/л, постпрандиальная гликемия 5,2 ммоль/л, С-пептид базальный 1,1 нг/мл, индекс HOMA-IR 0,75. Регулярная терапия 
Таблица 5. Переменные в дискриминантной модели

\begin{tabular}{lccc}
\hline \multicolumn{1}{c}{ Переменные } & Лямбда Уилкса & $\mathrm{F}$ & $\mathrm{p}$ \\
\hline ОХС & 0,870 & 9,887 & 0,002 \\
ТГ & 0,901 & 7,218 & 0,009 \\
Базальная гликемия & 0,899 & 7,401 & 0,008 \\
ХС ЛПНП/ХС ЛПВП & 0,866 & 10,243 & 0,002 \\
\hline
\end{tabular}

бисопрололом, лозартаном, ацетилсалициловой кислотой. Рандомизирована на терапию розувастатином 20 мг. Индивидуальное значение КЛДФ: $-2,633+0,214 \times 7,51+$ $+0,809 \times 1,15-0,252 \times 5,10+0,346 \times 3,39=-0,20777$. Исходя из величины КДЛФ, прогнозировалось отсутствие роста базальной гликемии в ходе терапии: ее уровень через 6 мес лечения составил 3,90 ммоль/л. Содержание постпрандиальной глюкозы, базального С-пептида и индекса НОМАIR составило 3,9 ммоль/л, 1,1 нг/мл и 1,36 соответственно.

Таким образом, полученные нами результаты демонстрируют возможность прогнозирования разнонаправленной динамики базальной гликемии при проведении 24-недельной липидснижающей терапии и идентификации тех пациентов, у которых в ходе этой терапии индивидуальный риск роста базальной гликемии является наиболее высоким.

\section{Нежелательные явления}

Переносимость терапии была хорошей, и все включенные пациенты закончили 24-недельный этап лечения. В ходе лечения неблагоприятные сердечно-сосудистые события отсутствовали, не было отмечено ни одного случая возрастания уровня в крови трансаминаз выше трех верхних границ и креатинфосфокиназы выше пяти верхних границ.

\section{ОБСУЖДЕНИЕ}

\section{Резюме результатов исследования}

На фоне 24-недельной терапии достоверный гиполипидемический эффект установлен при использовании всех трех режимов снижения ХС ЛПНП. Прием низких доз аторвастатина в сочетании с эзетимибом сопровождался возрастанием базальной гликемии уже через полгода терапии у 70,6\% пациентов и в среднем по группе. Хотя при титрации доз розувастатина и аторвастатина до целевого уровня ХС ЛПНП средние значения гликемии натощак существенно не изменялись, тем не менее, концентрации базальной глюкозы в процессе этой терапии повышались у 44,4\% и 48,5\% пациентов соответственно. В ходе пошагового многофакторного дискриминантного анализа установлено, что прогностической значимостью в отношении разнонаправленной динамики базальной гликемии в общей группе пациентов, получающих липидснижающую терапию, обладает комбинация исходных уровней в крови глюкозы натощак, ОХС, ТГ и соотношения ХС ЛПНП/ХC ЛПВП.

Обсуждение основного результата исследования

Хотя интенсивные режимы терапии статинами являются важнейшим условием эффективной профилактики атеросклеротических осложнений, ее проведение у опре-
Таблица 6. Нормированные коэффициенты канонической дискриминантной функции

\begin{tabular}{lc}
\hline \multicolumn{1}{c}{ Переменные } & Нормированные коэффициенты \\
\hline ОХС & 0,253 \\
ТГ & 0,513 \\
Базальная гликемия & $-0,642$ \\
ХС лПНП/ХС лПВП & 0,435 \\
\hline
\end{tabular}

деленной когорты пациентов сопряжено с развитием нежелательных диабетогенного и гипергликемического эффектов, механизм развития которых окончательно не установлен [3-6]. Нельзя исключить, что реализация этих эффектов с течением времени способна оказывать негативное влияние на состояние сосудистой стенки и потенциально снижать эффективность положительного воздействия статинов на сердечно-сосудистый прогноз, о чем сообщается в ряде наблюдательных когортных исследований [11]. В феврале 2012 г. Управлением по санитарному надзору за качеством пищевых продуктов и медикаментов (FDA, CША) издано сообщение о нежелательном эффекте статинов в отношении углеводного метаболизма в виде увеличения базальной гликемии и уровня гликированного гемоглобина [13]. Согласно рекомендациям экспертов Рабочей группы по безопасности статинов (Statin Diabetes Safety Task Force, 2014), при выявлении неблагоприятной динамики показателей углеводного обмена у пациентов, получающих липидснижающие препараты, рекомендована интенсификация сахароснижающей терапии [14].

До настоящего времени ни международные, ни национальные рекомендации не отдают каких-либо предпочтений выбору того или иного режима липидснижающей терапии с точки зрения его воздействия на обмен глюкозы/инсулина, в том числе и у пациентов с сочетанием метаболических факторов риска, хотя связь последних со статин-ассоциированными нарушениями метаболизма глюкозы считается установленной [3-6]. Нет ясности и в отношении характера воздействия на метаболизм глюкозы/ инсулина ингибитора кишечной абсорбции эзетимиба и его комбинации со статинами [7-10].

В ходе сравнения метаболических эффектов трех схем липидснижающей терапии мы получили данные о том, что различные режимы, индуцируя выраженное снижение уровня ХС ЛПНП, различались между собой по характеру динамики базальной гликемии. Так, под влиянием комбинации низких доз аторвастатина (средняя доза 13,5 мг/сут) с эзетимибом уже через полгода лечения у большинства

Таблица 7. Эффективность разработанной модели в прогнозировании роста гликемии на фоне липидснижающей терапии у пациентов с высоким и очень высоким сердечно-сосудистым риском

\begin{tabular}{ccccc}
\hline \multirow{2}{*}{$\begin{array}{c}\text { Гликемия рост (1)/ } \\
\text { нет (0) }\end{array}$} & \multicolumn{2}{c}{$\begin{array}{c}\text { Предсказанная } \\
\text { принадлежность } \\
\text { к группе }\end{array}$} & \\
\cline { 2 - 4 } & & 0 & 1 & \\
\hline \multirow{2}{*}{ Частотаго } \\
\cline { 3 - 4 } Исходные & 0 & 24 & 8 & 32 \\
\cline { 2 - 4 } & 1 & 9 & 27 & 36 \\
\hline & 0 & 75,0 & 25,0 & 100,0 \\
& 1 & 25,0 & 75,0 & 100,0 \\
\hline
\end{tabular}


пациентов и в среднем по группе имело место достоверное возрастание базальной гликемии, чего не происходило в группах розувастатина и аторвастатина. Литературные данные о влиянии эзетимиба и его сочетанного приема со статинами на обмен глюкозы/инсулина немногочисленны и весьма противоречивы [7-10]. Имеются единичные сообщения о благоприятном и нейтральном воздействии комбинированной терапии эзетимибом с правастатином или аторвастатином на чувствительность тканей к инсулину [10]. Тем не менее, в исследовании Moutzouri E и соавт. (2011) на фоне 12-недельной комбинированной терапии эзетимибом и симвастатином установлено возрастание степени инсулинорезистентности, что авторы связывали с неспособностью эзетимиба нейтрализовать нежелательные эффекты симвастатина в отношении метаболизма глюкозы/инсулина [8]. С последним наблюдением согласуются и полученные нами данные: реализацию гипергликемического эффекта комбинации аторвастатина и эзетимиба на фоне роста индекса инсулинорезистентности мы обнаружили через 24 нед от начала лечения. Одним из ключевых механизмов этих нежелательных метаболических сдвигов на фоне комбинированной терапии аторвастатином с эзетимибом, как было показано в нашем предыдущем исследовании, может являться дисбаланс секреции лептина и адипонектина [15]. Кроме того, нельзя исключить, что интенсивное снижение холестерина на фоне комбинированной липидснижающей терапии способно индуцировать увеличение концентрации глюкозы в крови посредством регуляции ее транспорта в клетки. Подавление под влиянием эзетимиба абсорбции холестерина в кишечнике на фоне ингибирования статинами ГМГ-КоА-редуктазы способно, по-видимому, дополнительно снизить активацию транспортеров глюкозы через холестерол-связывающие сайты.

Результаты нашего исследования продемонстрировали, что снижение уровня ХС ЛПНП на фоне других схем лечения, хотя в менее выраженной степени и в меньшем проценте случаев, также сопровождалось повышением концентрации базальной глюкозы крови, что на полугодовом этапе лечения обнаруживалось у почти половины пациентов при использовании средних доз аторвастатина и розувастатина.

Как известно, повышение гликемии натощак характеризует увеличение базальной продукции глюкозы печенью и отражает начальные этапы нарушения углеводного обмена. Следует отметить, что статистически значимых изменений содержания $\mathrm{HbA}_{1 с}$ в ходе 24-недельной липидснижающей терапии не определялось ни в одной из групп. Тем не менее, это не исключает, что при более длительных сроках липидснижающей терапии возможно усугубление статин-ассоциированных нарушений обмена глюкозы/инсулина с возрастанием уровня $\mathrm{HbA}_{1 c}$. Так, в наших предыдущих исследованиях установлено, что при длительности приема аторвастатина в низких и средних дозах от 36 нед имел место значимый рост не только базальной гликемии, но и базального и постпрандиального уровня С-пептида, а также содержания $\mathrm{HbA}_{1 c}$ [16].

Математическое моделирование широко используется для повышения эффективности первичной и вторичной профилактики СД, а также для разработки и оценки эффективности лекарственных средств и процедур [17]. С целью определения комбинации факторов, способных прогнози- ровать характер изменений базальной гликемии на фоне 24-недельной липидснижающей терапии, в нашем исследовании был выполнен многофакторный дискриминантный анализ в общей группе пациентов, то есть при использовании всех режимов снижения ХС ЛПНП. В каноническую линейную дискриминантную функцию было включено 4 фактора: исходные уровни базальной глюкозы, ОХС, ТГ и соотношение ХС ЛПНП/ХС ЛПВП. Предложенная диагностическая модель помогает предсказать индивидуальный риск возрастания гликемии при снижении уровня ХС ЛПНП под влиянием липидснижающей терапии, что представляется объективной необходимостью у больных с наличием метаболических факторов риска.

Наши данные демонстрируют, что хотя степень воздействия разных режимов снижения уровня ХС ЛПНП на уровень глюкозы натощак различается, тем не менее, любая из схем липидснижающей терапии может сопровождаться ростом базальной гликемии в случае сочетания нескольких метаболических факторов риска.

Исходя из полученной нами модели, важное прогностическое значение в отношении роста гликемии имеет состояние липидтранспортной функции крови. При сочетании высоких уровней ОХС, ХС ЛПНП и повышенного содержания в крови триглицеридов требуется назначение пропорциональных степени этого повышения доз статинов. Чем выше доза статина, тем сильнее выражен эффект ингибирования ГМГ-КоА-редуктазы, что, в свою очередь, сопровождается более выраженным подавлением синтеза изопреноидов, снижением экспрессии ГЛЮТ 4 и обратного захвата глюкозы. Как установлено в недавнем метаанализе генетических исследований D.Swerdlow и соавт. (2014), диабетогенный эффект статинов, по крайней мере частично, объясняется их основным механизмом действия - ингибированием ГМГ-КоА-редуктазы [18]. Полученные нами данные согласуются с точкой зрения о связи статин-ассоциированных нарушений метаболизма глюкозы/инсулина непосредственно с их гиполипидемическим действием [19] и подтверждают результаты исследований других авторов, показавших, что при наличии не более одного метаболического фактора риска даже на фоне высокой дозы аторвастатина частота новых случаев СД не увеличивается, тогда как в случае сочетания нескольких факторов риска встречаемость ассоциированного с приемом аторвастатина СД возрастает на 24\% [20]. Результаты нашей работы находятся в соответствии с данными ряда крупных рандомизированных клинических исследований, показавших связь роста новых случаев СД с исходными метаболическими нарушениями, в том числе с повышенным уровнем триглицеридов [21], и поддерживают предположение о существовании тесной физиологической взаимосвязи обратной направленности между уровнем ХС ЛПНП и гликемией [22-23]. Совсем недавно получено новое подтверждение этой гипотезы: установлено, что ассоциация ряда полиморфизмов гена PCSK9 со сниженным уровнем ХС ЛПНП имеет также взаимосвязь с повышенными значениями базальной гликемии, массы тела и отношения окружности талии к окружности бедер [24].

\section{Ограничения исследования}

Возможными ограничениями исследования являются открытый дизайн, использование небольших доз статинов, преобладание женщин, построение прогностической 
модели на основе оценки динамики базальной гликемии под влиянием разных схем липидснижающей терапии без учета потенциальных различий метаболических эффектов того или иного режима снижения ХС ЛПНП. В нашем исследовании фоновая терапия у большинства пациентов включала кардиоселективные бета-адреноблокаторы и тиазидные диуретики, что могло иметь дополнительное влияние на обмен глюкозы/инсулина. Данные пациентов, получавших терапию инсулином (13,2\% от общего числа включенных пациентов), были исключены из анализа динамики индекса HOMA-IR.

\section{ЗАКЛЮЧЕНИЕ}

В нашей работе впервые сделана попытка установить, какая именно комбинация исходных метаболических факторов риска способна прогнозировать характер изменений базальной гликемии в ходе длительной липидснижающей терапии. Созданная нами диагностическая модель позволяет определить индивидуальную вероятность ассоциированного со снижением ХС ЛПНП роста базальной гликемии с помощью определения в исходном состоянии четырех лабораторных показателей - базальной гликемии, содержания в крови общего холестерина, триглицеридов и соотношения между концентрацией холестерина антиатерогенных и атерогенных классов липопротеинов. Пациентов с сочетанием метаболических факторов риска, име- ющих высокую вероятность реализации нежелательных эффектов липидснижающей терапии в отношении гликемии, следует информировать о возможности их возникновения, рекомендовать отказ от курения, контроль и снижение массы тела, окружности талии, регулярные физические нагрузки и мониторинг уровня глюкозы. Для верификации разработанной нами формулы необходимы дальнейшие исследования механизмов статин-ассоциированных нарушений обмена глюкозы/инсулина и поиск методов их профилактики у пациентов из группы риска.

\section{ДОПОЛНИТЕЛЬНАЯ ИНФОРМАЦИЯ}

Финансирование работы. Работа выполнена на основе бюджетного финансирования: государственное задание 2016-2018 гг, АААА-А15-115123110026-3 от 31.12.2015, № 0548-2014-0018, шифр темы 030.

Конфликт интересов. Авторы декларируют отсутствие явных и потенциальных конфликтов интересов, связанных с публикацией настоящей статьи

Участие авторов. О.А. Кошельская, Т.Е. Суслова, Р.С. Карпов - концепция и дизайн исследования, редактирование и окончательное утверждение статьи; А.С. Сушкова - ведение пациентов, создание базы данных; А.С. Сушкова, О.А. Журавлева, И.В. Винницкая, Е.С. Кравченко - анализ и интерпретация данных; О.А. Кошельская, А.С. Сушкова, О.А. Журавлева, И.В. Винницкая - написание текста статьи; Е.С. Кравченко - определение лабораторных показателей; Н.Г. Бразовская - статистический анализ данных, редактирование статьи

\section{СПИСОК ЛИТЕРАТУРЫ | REFERENCES}

1. Catalano AL, Graham I, De Backer G, et al. 2016 ESC/EAS Guidelines for the management of dyslipidaemias. Eur Heart J. 2016;37(39):29993058. doi: 10.1093/eurheartj/ehw272

2. Аронов Д.М., Арабидзе Г.Г., Ахмеджанов Н.М., и др. Диагностика и коррекция нарушений липидного обмена с целью профилактики и лечения атеросклероза. Российские рекомендации, V пересмотр // Российский кардиологический журнал. - 2012. - Т.96. №5 - C. 1-32. [Aronov DM, Arabidze GG, Akhmedzhanov NM, et al. Diagnosis and correction of lipid disorders for the prevention and treatment of atherosclerosis. Russian recommendations. $\vee$ revision Russian Cardiology Journal. 2012;96(5):1-32. (In Russ.)]

3. Sattar N, Preiss D, Murray HM, et al. Statin and risk of incident diabetes: a collaborative meta-analysis of randomized statin trials. Lancet. 2010;375(9716): 735-742. doi: 10.1016/\$0140-6736(09)61965-6

4. Chen CW, Chen TC, Huang KY, et al. Differential impact of statin on new-onset diabetes in different age groups: a population-based case-control study in women from an Asian country. PLoS One. 2013;8(8):e71817. doi:10.1371/journal.pone.0071817

5. Mora S, Glynn RJ, Hsia J, et al. Statins for the primary prevention of cardiovascular events in women with elevated high-sensitivity C-reactive protein or dyslipidemia: results from the Justification for the Use of Statins in Prevention: An Intervention Trial Evaluating Rosuvastatin (JUPITER) and meta-analysis of women from primary prevention trials. Circulation. 2010;121(9): 1069-1077. doi: 10.1161/circulationaha.109.906479

6. Shen L, Shah BR, Reyes EM, et al. Role of diuretics, $\beta$ blockers, and statins in increasing the risk of diabetes in patients with impaired glucose tolerance: reanalysis of data from the NAVIGATOR study. BMJ. 2013;347:f6745. doi:10.1136/bmj.f6745

7. Ballantyne CM, Houri J, Notarbartolo A et al. Effect of ezetimibe coadministered with atorvastatin in 628 patients with primary hypercholesterolemia: a prospective, randomized, double-blind trial. Circulation. 2003;107(19):2409-2415. doi: 10.1161/01.cir.0000068312.21969.c8

8. Moutzouri E, Liberopoulos E, Mikhailidis DP, et al. Comparison of the effects of simvastatin vs. rosuvastatin vs. simvastatin/ezetimibe on parameters of insulin resistance. Int Clin Pract. 2011;65(11):1141-1148. doi: 10.1016/s1567-5688(11)70080-0
9. Takeshita Y, Takamura T, Honda M, et al. The effects of ezetimibe on non-alcoholic fatty liver disease and glucose metabolism: a randomised controlled trial. Diabetologia. 2014;57(5):878-890. doi: 10.1007/s00125-013-3149-9

10. Her AY, Kim JY, Kang SM, et al. Effects of atorvastatin 20 mg, rosuvastatin $10 \mathrm{mg}$, and atorvastatin/ezetimibe $5 \mathrm{mg} / 5 \mathrm{mg}$ on lipoproteins and glucose metabolism. J Cardiovasc Pharmacol Ther. 2010;15(2):167-174. doi: 10.1177/1074248409357922

11. Wang KL, Liu CJ, Chao TF, et al. Statins, risk of diabetes, and implications on outcomes in general population. J Am Coll Cardiol. 2012;60(14):1231-1238. doi: 10.1016/j.jacc.2012.05.019

12. Кухарчук В.В., Сусеков А.В., Зубарева М.Ю., и др. Диагностика и коррекция нарушений липидного обмена с целью профилактики и лечения атеросклероза. Российские рекомендации. IV пересмотр // Кардиоваскулярная терапия и профилактика. - 2009. T. 8. - №6. - C. 3-58. [Kukharchuk WV, Susekov AV, Zubareva M, et al. Diagnosis and correction of lipid disorders for the prevention and treatment of atherosclerosis. Russian recommendations. IV revision. Cardiovascular Therapy and Prevention. 2009;8(6):3-58. (In Russ.)]

13. U.S. Food and Drug Administration [Internet]. FDA Drug Safety Communication. Important safety label changes to cholesterol lowering statin drugs. 2012 [cited 2017 Mar 18]. Available from: http://www. fda.gov/Drugs/DrugSafety/ucm293101.htm.

14. Maki KC, Ridker PM, Brown WV, et al. An assessment by the Statin Diabetes Safety Task Force: 2014 update. J Clin Lipidol. 2014;8(S3):17-29. doi: 10.1016/j.jacl.2014.02.012

15. Кошельская О.А., Винницкая И.В., Конько Т.Ю., и др. Сравнительное рандомизированное исследование по оценке влияния длительной терапии розувастатином в сочетании с комбинацией аторвастатина и эзетимиба на показатели углеводного обмена и уровень адипокинов у больных ишемической болезнью сердца и сахарным диабетом // Кардиология. - 2015. - Т. 55. - № 3. C. 67-74. [Koshelskaya OA, Vinnitskaya IV, Konko TYu, et al. Comparative Randomized Study of the Effects of Long-Term Therapy With Rosuvastatin and With Combination of Atorvastatin and Ezetimibe on Carbohydrate Metabolism and Adipokynes Levels in Patients With Coronary Artery Disease and Diabetes Mellitus. Kardiologiia. 2015;55(3):67-74. (In Russ.)] doi: 10.18565/cardio.2015.3.67-74 
16. Кошельская О.А., Сушкова А.С., Суслова Т.Е., и др. Длительная терапия аторвастатином у больных ишемической болезнью сердца и сахарным диабетом: влияние на гликемию и локальную сосудистую жесткость // Сердие: журнал для практикующих врачей. - 2013. T. 12. - № 4. - C. 223-229. [Koshel'skaya O.A., Sushkova A.S., Suslova T.E. et al. Long-term atorvastatin therapy in patients with ischemic heart disease: effect on glycemia and local vascular stiffness. Russian Heart Journal. 2013;12(4):223-229. (In Russ.)] doi: 10.18087/rhj.2013.4.1820

17. Карпельев В.А., Филиппов Ю.И., Тарасов Ю.В., и др. Математическое моделирование системы регуляции гликемии у пациентов с сахарным диабетом // Вестник Российской Академии Медииинских Наук. - 2015. - Т. 70. - №5. - C. 549-560. [Karpel'ev VA, Filippov Yul, Tarasov YuV, et al. Mathematical Modeling of the Blood Glucose Regulation System in Diabetes Mellitus Patients. Annals of the Russian Academy of Medical Sciences. 2015;70(5):549-560. (In Russ.)] doi: 10.15690/vramn.v70.15.1441.

18. Swerdlow DI, Preiss D, Kuchenbaecker KB, et al. HMG-coenzyme A reductase inhibition, type 2 diabetes, and bodyweight: evidence from genetic analysis and randomised trials. Lancet. 2015;385(9965):351361. doi: 10.1016/S0140-6736(14)61183-1
19. Zaharan NL, Swerdlow DI, Bennet K. Statins and risk of treated incident diabetes in a primary care population. Br J Clin Pharmacol. 2013;75(4):1118-1124. doi: 10.1111/j.1365-2125.2012.04403.x

20. Waters DD, Ho JE, Boekholdt SM, et al. Cardiovascular event reduction versus new-onset diabetes during atorvastatin therapy: effect of baseline risk factors for diabetes. J Am Coll Cardiol. 2013;61(2):148-152. doi: 10.1016/j.jacc.2012.09.042

21. Waters DD, Ho JE, DeMicco DA, et al. Predictors of new-onset diabetes in patients treated with atorvastatin: results from 3 large randomized clinical trials. J Am Coll Cardiol. 2011;57(14):1535-1545. doi: 10.1016/j.jacc.2010.10.047

22. Besseling J, Kastelein JJ, Defesche JC, et al. Association between familial hypercholesterolemia and prevalence of type 2 diabetes mellitus. JAMA. 2015;313(10):1029-1036. doi: 10.1001/jama.2015.1206

23. White J, Swerdlow DI, Preiss D, et al. Association of lipid fractions with risks for coronary artery disease and diabetes. JAMA Cardiol. 2016;1(6):692-699. doi: 10.1001/jamacardio.2016.1884

24. Thompson G. Limitations of cholesterol lowering with PCSK9 inhibitors. Lancet Diabetes Endocrinol. 2017;5(4):241-243. doi: 10.1016/s2213-8587(17)30060-8

\section{ИНФОРМАЦИЯ ОБ АВТОРАХ [AUTHORS INFO]}

Кошельская Ольга Анатольевна, д.М.Н., профессор [Olga A. Koshelskaya, MD, PhD, Professor]; адрес: 634012, Томск, ул. Kиевская, д. 111A [address: 111A Kievskaya str., 634012 Tomsk, Russian Federation]; телефон: 8 (3822) 55-84-91; ORCID: http://orcid.org/0000-0002-6679-1269; eLibrary SPIN: 3093-4903; e-mail: koshel@live.ru.

Сушкова Анастасия Сергеевна [Anastasiya S. Sushkova, MD]; ORCID: http://orcid.org/0000-0003-4904-869X; eLibrary SPIN: 3014-3119; e-mail: nastya_sush@mail.ru. Журавлева Ольга Александровна, К.м.н. [Olga A. Zhuravleva, MD, PhD]; ORCID: http://orcid.org/0000-0003-0106-6813; eLibrary SPIN: 9749-8912; e-mail: olgazh.cardio@mail.ru. Винницкая Ирина Владимировна [Irina V. Vinnizkaya, MD]; ORCID: http://orcid.org/0000-0002-6489-0650; eLibrary SPIN: 3879-3072; e-mail: irina_khor@list.ru. Бразовская Наталия Георгиевна, к.м.Н., доцент [Nataliya G. Brazovskaya, MD, PhD, associate professor]; ORCID: http:// orcid.org/0000-0002-0706-9735; eLibrary SPIN: 3410-2015; e-mail: brang@mail.ru. Кравченко Елена Сергеевна [Elena S. Kravchenko, MD]; ORCID: http://orcid. org/0000-0002-1235-9956; eLibrary SPIN: 9385-8321; e-mail: nikonova@cardio-tomsk.ru. Суслова Татьяна Евгеньевна, K.M.н. [Tatyana E. Suslova, MD, PhD]; ORCID: http://orcid.org/0000-0001-9645-6720; eLibrary SPIN: 9588-9414; e-mail: tes@cardio-tomsk.ru. Карпов Ростислав Сергеевич, д.м.н., профессор, академик PAH [Rostislav S. Karpov, MD, PhD, Professor]; ORCID: http://orcid.org/0000-0002-7011-4316; eLibrary SPIN: 8263-2641; e-mail: tvk@cardio-tomsk.ru.

\section{ЦИТИРОВАТЬ:}

Кошельская О.А., Сушкова А.С., Журавлева О.А., Винницкая И.В., Бразовская Н.Г., Кравченко Е.С., Суслова Т.Е., Карпов Р.С. Прогнозирование динамики базальной гликемии в ходе полугодовой липидснижающей терапии у пациентов высокого сердечно-сосудистого риска // Сахарный диабет. - 2017. T. 20. — №5. - C. 374-383. doi: 10.14341/DM8633

\section{TO CITE THIS ARTICLE:}

Koshelskaya OA, Sushkova AS, Zhuravleva OA, Vinnizkaya IV, Brazovskaya NG, Kravchenko ES, Suslova TE, Karpov RS. Prediction of basal glycaemia dynamics during treatment with 6-month lipid-lowering therapy in patients at high risk of cardiovascular disease.. Diabetes mellitus. 2017;20(5):374-383. doi: 10.14341/DM8633 